\title{
A Robotic Experiment Toward Understanding Human Gas-Source Localization Strategies
}

\author{
Andres Gongora, Javier G. Monroy and Javier Gonzalez-Jimenez \\ Machine Perception and Intelligent Robotics group (MAPIR) \\ Departamento de Ingeniería de Sistemas y Automática, and \\ Instituto de Investigación Biomédica de Málaga (IBIMA) \\ Universidad de Málaga, Spain \\ Email: andresgongora@uma.es, jgmonroy@uma.es, javiergonzalez@uma.es
}

\begin{abstract}
This paper describes an experiment for gas-source localization with a human-teleoperated mobile robot devised to gather data on how humans search for odor-sources. To that end, more than 150 repetitions of the search process are recorded for 69 test subjects, under 4 sensor configurations (including electronic nose, anemometer and video camera) and 4 scenarios (i.e. with different wind-flow conditions and gas-source position). The experiment has been carried out with a ROS-based simulator that allows driving the robot while recording data of interest (e.g. driving commands, robot localization, sensor measurements, groundtruth, etc.) for further analyzing the human process of gas-source searching, and computational fluid dynamics (CFD) to generate realistic and repeatable test conditions. The manuscript describes the different environmental parameters and sensor combinations of the experiment, and explains the methodology under which it was executed. The obtained dataset is publicly available at http://mapir.isa.uma.es/mapirwebsite/index.php/253-gsl-dataset.
\end{abstract}

\section{INTRODUCTION}

Robotic olfaction is the discipline that brings together artificial olfaction and mobile robotics. It involves equipping robots with small and portable electronic-noses (e-noses) [1] in order to extend their sensory capabilities with the perception of volatile substances [2], [3]. The relevance of these "olfactory robots" lies not only in their usefulness for a wide range of potential odor-related applications [4] [5], but also in the boost that the sense of smell brings towards the design of more intelligent robotic behaviours.

One of the most relevant tasks for olfactory robots is the automatic localization of a gas emission source. Yet this is still an open issue, with solutions that either make strong simplifications about how gas spreads [6], or rely on unrealistic assumptions, such as homogeneous wind fields [7] or the absence of obstacles in the environment [8]. Advances in this challenging problem encounter two main hurdles: the lack of reliable experimental data to develop, validate, and compare new approaches, and the still limited knowledge on the search strategy that we (and animals in general) have learned through evolution to find odor sources.

Intuitively, the combination of smell with other sensory inputs (e.g. vision, perceived wind flow, etc) is key for human gas-source localization, but we still lack of a quantification of their benefits and insight on the underlying mechanism. Moreover, we suspect that this process may not only rely on the perceived information, but also on learned sensorymotor patterns (like those revealed by psychologists in the "kitten carousel" experiment for visual inputs [9]), which would add a reactive component to the search strategy. This manuscript presents an experiment intended to collect new data to advance toward these studies. The experiment considers therefore several sensor combinations, along artificial olfaction, and employs a realistic simulator to solve the problem of controlling the environmental conditions (i.e gas distribution and wind conditions). The result of this work is a dataset that contains over 150 repetitions of the experiment from 69 participants, and is intended for the future analysis of relevant questions, including whether is possible to learn from humans how to search for gas-sources in unknown environments.

\section{DESCRIPTION}

The objective of this work is to gather data of how humans search and localize gas-emission sources, that is, to record how humans move towards an odor-source depending on the information they perceive. To that end we asked students at our campus to participate in a research experiment where they had to locate, as fast and accurately as possible, a gas-leak with a teleoperated mobile robot in a simulated environment. For each session, we logged the environmental parameters, the robot state-variables (odometry, pose, speed, etc.) and distance to the source, the measurements of the sensors on board the robot, and the user-input commands.

Next, we describe the simulation environment, the different sensors that the robot carried, and the method in which the experiment was carried out.

\section{A. Simulation Setup}

The presented experiment has been carried out with the help of simulation tools because of the need to repeat the searching process several times while maintaining identical environmental conditions (wind flow, gas release, plume shape, etc). This, which is mandatory for the proper analysis of the data, its hardly achievable in real-world setups. Nevertheless, it must be stressed that the relevant data in this work is not that provided by the simulator, but those parameters and variables related to the user activity during the searching process. 
Concretely, we developed our simulator with ROS [10] and RVIZ [11], and employed computational fluid dynamics (CFD) [12] to generate dynamic and realistic gas dispersions in a complex 3D indoor environment, including gas-plumes, eddies, vortices and turbulences in four different wind-flow regimens (see Fig. 1). Note that the gas dispersions were partly preprocessed to reduce run-time computation.

\section{B. Sensory Configurations}

Part of the experiment consists in providing the user with different amounts of information to observe how their search efficiency is affected. Specifically, we tested four configurations of cumulative sensors and HUD overlays (see Fig. 2 ).

- Configuration 1: It represents the basic setup containing the minimum number of sensors to accomplish the search task. Concretely, the robot is only equipped with a frontal camera (for navigation purposes) and with a gas sensor to measure the gas concentration. The latter is presented to the operator both as a visual bar (Fig. 2a) and as an audible signal (analogous to a Geiger counter).

- Configuration 2: It extends the basic setup with the incorporation of a 2D anemometer measuring the horizontal wind flow. It is intended to help determine the average wind direction, and is presented to the operator as an arrow with direction and size relative to the sensed wind flow (Fig. 2b).

- Configuration 3: It exploits the spatio-temporal information the operator gathers while searching for the gasleak. That is, this configuration provides a real-time gas distribution map [13] that is continuously updated from the gas concentration measurements (Fig. 2c).

- Configuration 4: This last setup adds visual information in the form of potential gas-source candidates that can be seen with the frontal camera (Fig. 2a), and represents a case scenario where the operator has prior knowledge about the gas source (shape, size and color).

\section{Method}

All participants were students from our university campus. After informing them of their goal, to park the teleoperated robot as close as possible to where they believed the gas-leak was located, we handed them leaflets with specific instructions and an explanation of the robot's sensors.

As for operating the simulator, we provided the users with a keyboard to drive the robot, a screen to visualize the robot's camera and navigation map, and a headset for the gas sensor's audible signal. Each experiment started with the robot at the same location regardless of the simulated scenario (selected at random) and active sensors. Also, the initial gas distribution was fast-forwarded to a semi-stationary state (100 seconds after initial gas release), ensuring that it had extended to all affected rooms.

Finally, the experiments ended at the user's request once he believed he had located the gas-leak. Note that volunteers who wished to participate more than once did so for different scenarios and sensor configurations.

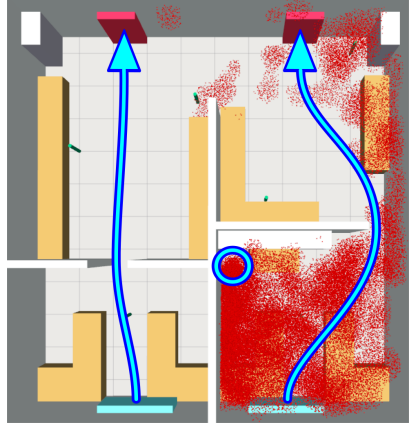

(a) Scenario 1

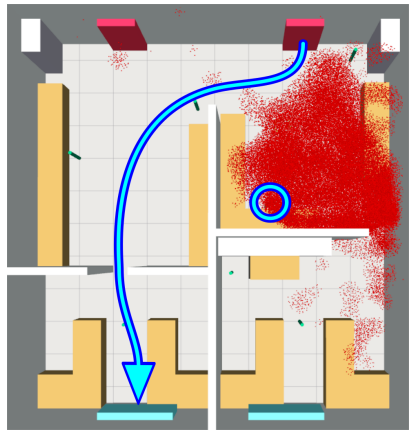

(c) Scenario 3

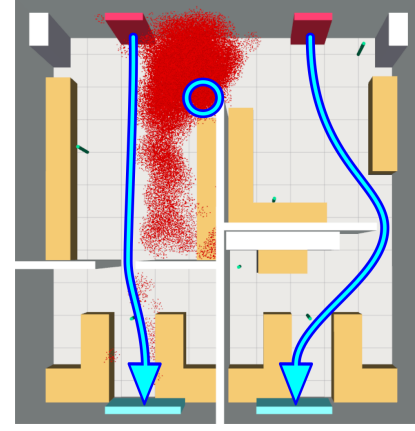

(b) Scenario 2

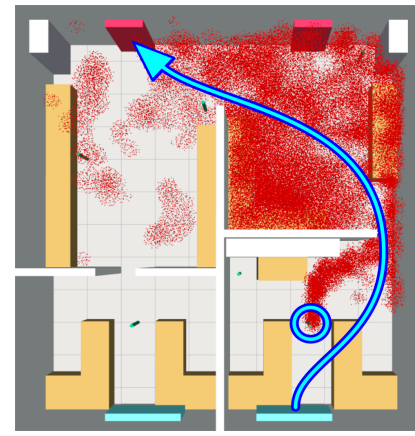

(d) Scenario 4
Figure 1: The four scenarios employed for the experiments (after 100 seconds) showing the wind's main flow (arrows), the location of the active gas-leak (circles), the other gas-leak candidates (green cylinders), and the simulated gas distribution (point-cloud) with concentrations of up to $25 \mathrm{ppm}$.

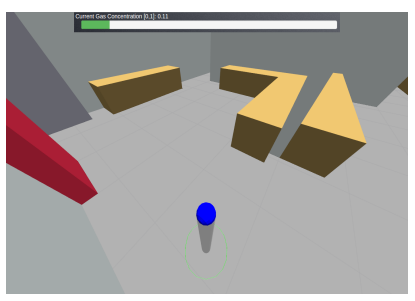

(a) Configuration 1

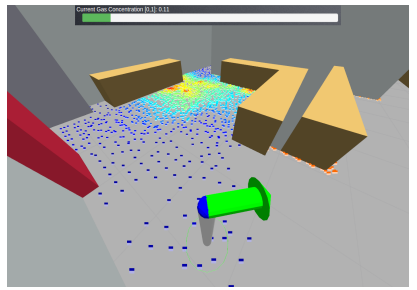

(c) Configuration 3

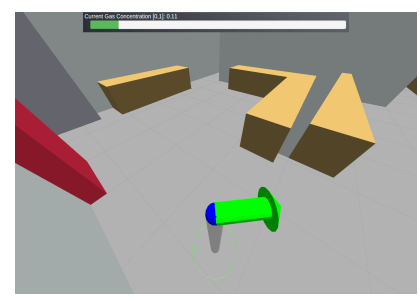

(b) Configuration 2

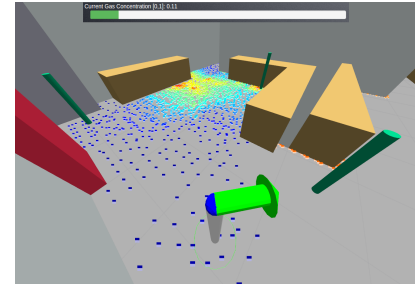

(d) Configuration 4
Figure 2: Representation of the four sensor configurations as presented to the human operator (navigation map omitted). (a) Presents the user with the gas concentration at the current location (notice the top bar), (b) adds wind information, (c) inserts a false color gas distribution map at floor level, and (d) indicates all possible gas leak candidates. 


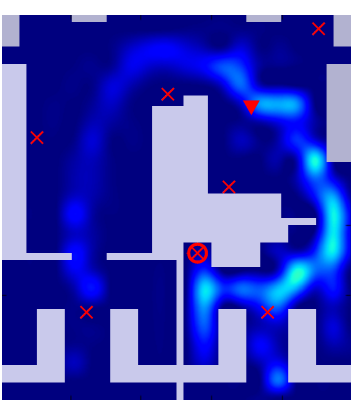

(a) Olfaction only

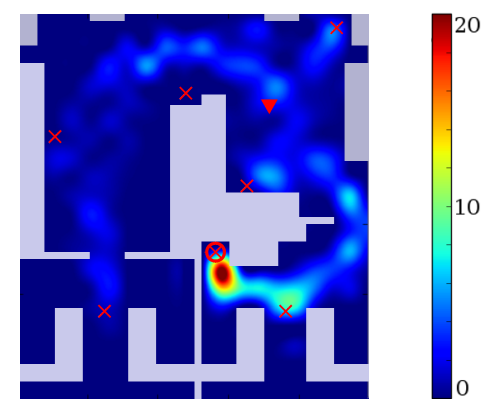

(b) Candidate visualization
Figure 3: Average location of the robot in Scenario 1 (measured in seconds and smoothed with a $0.5 \mathrm{~m}$ radius) comparing sensor configuration 1 (olfaction only) against configuration 4 (gas-leak candidate visualization). The triangle indicates the robot's starting position and the crosses the possible gas-leaks, of which the encircled one is the active source.

\section{DATA}

The result of the experiments is a dataset that includes 160 recordings from 69 volunteers (54 males, 15 females) distributed between the four scenarios and the four sensor combinations as shown in Table I. It is available for download at our webpage [14] in rosbag [10] and CSV file format.

As an example of the gathered data, Fig. 3 shows a map of the robot's average location in Scenario 1 for sensory configurations 1 and 4, showing how the average behaviour of the users changes from a general exploration, when presented with oflactory information only (Fig. 3a), to a more forward approach towards the visible gas-leak candidates (Fig. 3b). Also by way of illustration, Fig. 4 shows for scenario 1 how the average distance from the robot to the gas-leak decreases over time for all sensor configurations as the users consecutively approached the gas leak.

\section{CONCLUSIONS AND FUTURE WORK}

This work described an experiment intended to gather the necessary data to understand human gas-source localization. It employed a CFD simulator to create replicable and realistic scenarios on which humans had to find a gas-leak with a mobile robot. The result of the experiment is a dataset with 160 search samples and 16 test configurations.

In this line, our next steps will focus on analyzing the gathered data to extract conclusions regarding each sensor's efficiency and the strategies that the participants employed, and try to develop a new bio-inspired algorithm based on them.

Table I: Number of experiments for the different scenarios and sensory configurations.

\begin{tabular}{|c|c|c|c|c|}
\cline { 2 - 5 } \multicolumn{1}{c|}{} & Conf. 1 & Conf. 2 & Conf. 3 & Conf. 4 \\
\hline Scenario 1 & 9 & 9 & 11 & 9 \\
\hline Scenario 2 & 10 & 9 & 9 & 11 \\
\hline Scenario 3 & 9 & 10 & 10 & 11 \\
\hline Scenario 4 & 12 & 10 & 13 & 8 \\
\hline
\end{tabular}

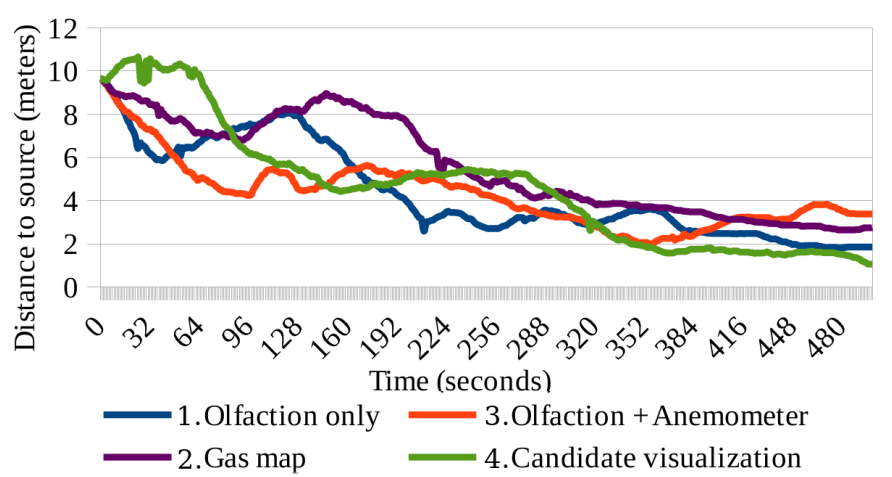

Figure 4: Average distance to the gas source over time for each sensory configuration in Scenario 1, shown for the first 500 seconds (aproximately 90\% of the experiments finish).

\section{ACKNOWLEDGMENT}

This work has been funded by Governments of Spain and Andalusia, and the European Regional Development Fund under project TEP530.

\section{REFERENCES}

[1] C. Sanchez-Garrido, J. G. Monroy, and J. Gonzalez-Jimenez, "A configurable smart e-nose for spatio-temporal olfactory analysis," IEEE SENSORS 2014 Proceedings, pp. 1968-1971, 2014.

[2] A. Loutfi, S. Coradeschi, A. J. Lilienthal, and J. Gonzalez, "Gas distribution mapping of multiple odour sources using a mobile robot," Robotica, vol. 27, no. 02, pp. 311-319, 2009.

[3] V. Hernandez Bennetts, E. Schaffernicht, V. Pomareda, A. J. Lilienthal, S. Marco, and M. Trincavelli, "Combining non selective gas sensors on a mobile robot for identification and mapping of multiple chemical compounds," Sensors, vol. 14, no. 9, pp. 17331-17352, 2014.

[4] J. Norberto Pires, C. Distante, G. Indiveri, and G. Reina, "An application of mobile robotics for olfactory monitoring of hazardous industrial sites," Industrial Robot: An Int. Journal, vol. 36, no. 1, pp. 51-59, 2009.

[5] J. G. Monroy, J. Gonzalez-Jimenez, and C. Sanchez-Garrido, "Monitoring household garbage odors in urban areas through distribution maps," in IEEE SENSORS 2014 Proceedings. IEEE, 2014, pp. 1364-1367.

[6] M. Saska, J. Langr, and L. Přeučil, "Plume tracking by a self-stabilized group of micro aerial vehicles," in International Workshop on Modelling and Simulation for Autonomous Systems. Springer, 2014, pp. 44-55.

[7] G. Ferri, E. Caselli, V. Mattoli, A. Mondini, B. Mazzolai, and P. Dario, "SPIRAL: A novel biologically-inspired algorithm for gas/odor source localization in an indoor environment with no strong airflow," Robotics and Autonomous Systems, vol. 57, no. 4, pp. 393-402, 2009.

[8] Y. Wada, H. Matsukura, and H. Ishida, "Estimation of gas source location from fluctuating readings of gas sensors and anemometer on mobile robot in outdoor environment," ECS Transactions, vol. 75, no. 16, pp. 99-106, 2016.

[9] R. Held and A. Hein, "Movement-produced stimulation in the development of visually guided behavior." Journal of comparative and physiological psychology, vol. 56, no. 5, p. 872, 1963.

[10] M. Quigley, K. Conley, B. P. Gerkey, J. Faust, T. Foote, J. Leibs, R. Wheeler, and A. Y. Ng, "ROS: an open-source Robot Operating System," in ICRA Workshop on Open Source Software, 2009.

[11] "RVIZ: a 3d visualizer for displaying sensor data and state information from ROS," http://wiki.ros.org/rviz, accessed: 2017-01-09.

[12] "OpenFOAM: a open source cfd toolbox," www.openfoam.org, accessed: 2017-01-09.

[13] J. G. Monroy, J.-L. Blanco, and J. González-Jiménez, "Time-variant gas distribution mapping with obstacle information," Autonomous Robots, vol. 40 , no. 1 , pp. $1-16,2016$.

[14] "Dataset and related media hosting site." [Online]. Available: http://mapir.isa.uma.es/mapirwebsite/index.php/253-gsl-dataset 